\title{
EXTENDED PIECEWISE MARKOV PROCESSES IN CONTINUOUS TIME
}

\section{DEFINITIONS AND NOTATION}

In this paper stationary extended piecewise Markov processes on a general state space are considered. Analogical processes on a discrete state space were introduced in [9] as a natural extension of piecewise Markov processes defined in [10].

For these processes, relations between the stationary probability distribution of the process and the stationary probability distributions of some imbedded Markor chains are given. These relations are obtained by using an extension of the process to a Markov process and contractionsemi-group theory.

Applications of the obtained results to an investigation of queueing systems with breakdown of the service line are also given in this paper.

Let us introduce the notation. obligatory in the whole paper: $\mathscr{R}=$ $=(-\infty, \infty), \mathscr{R}_{+}=(0, \infty), \mathscr{R}_{0}=[0, \infty), \mathscr{B}_{+}$and $\mathscr{B}_{0}$ stand for the $\sigma$-algebras of Borel subsets of $\mathscr{R}_{+}$and $\mathscr{R}_{0}$, respectively, and $\mathscr{B}_{1}$ denotes the $\sigma$-algebra of all subsets of the set $\{0,1\}$. Let $\mathscr{X}$ be a complete subset of $\mathscr{R}$ and let $\mathscr{F}$ be the $\sigma$-algebra of Borel subsets of $\mathscr{X}$.

Let us assume that on the measurable space $(\mathscr{X}, \mathscr{F})$ there are given: a set of measurable Markov transition functions

$$
\left\{P_{a, y}(t, x, A), t \geqslant 0, \dot{x} \in \mathscr{X}, A \in \mathscr{F}\right\}, \quad a=0,1, y \in \mathscr{X}
$$

(i.e., for every $a, t, x, y, P_{a, y}(t, x, \cdot)$ is a probability measure and, for every $a, A, P_{a, \cdot}(\cdot, \cdot, A)$ is a $\left(\mathscr{B}_{0} \times \mathscr{F}^{2}\right)$-measurable function);

two stochastic kernels $\left\{Q_{a}(x, A), x \in \mathscr{X}, A \in \mathscr{F}\right\}, a=0,1$ (i.e., for every $a, x, Q_{a}(x, \cdot)$ is a probability measure and, for every $a, A, Q_{a}(\cdot, A)$ is an $\mathscr{F}$-measurable function);

a set of probability measures $\left\{\mu_{a, y}(C), C \in \mathscr{B}_{0}\right\}, a=0,1, y \in \mathscr{X}$, such that, for every $y, \mu_{1, y}(\{0\})=0$. Definition 1 . A stochastic process $\{X(t), t \geqslant 0\}$ defined on the
probability space $(\Omega, \sigma, \operatorname{Pr})$, valued in the state space $(\mathscr{X}, \mathscr{F})$, whose 
trajectories are right-hand continuous functions and for which the lefthand limits exist is said to be an extended piecewise Markov process if the following conditions (a)-(d) are fulfilled:

(a) There exists a sequence of random variables $0=\tau_{0} \leqslant \tau_{1}<\tau_{2}$ $\leqslant \tau_{3}<\ldots$ defined on $(\Omega, \sigma, \operatorname{Pr})$, valued in $\left(\mathscr{R}_{0}, \mathscr{B}_{0}\right)$, such that the process

$$
\left\{X(t), \tau_{2 m+a} \leqslant t<\tau_{2 m+a+1}\right\}, \quad a=0,1, m=0,1, \ldots,
$$

is a homogeneous Markov process with the transition function $P_{a, y}(t, x, A)$, dependent on $a$ and on the condition $\left\{X\left(\tau_{2 m+a}\right)=y\right\}$.

(b) At the moments $\tau_{2 m+a}, a=0,1, m=0,1, \ldots$, for which $\tau_{2 m+a} \neq \tau_{2 m+a+1}$ the process changes the state jumping with a transition probability $Q_{1-a}(x, A)$.

(c) At the moments $\tau_{2 m}, m=0,1, \ldots$, for which $\tau_{2 m}=\tau_{2 m+1}$ the process changes the state jumping with a transition probability equal to

$$
\int_{\mathscr{X}} Q_{1}(x, d y) Q_{0}(y, A) \text {. }
$$

(d) For every $a=0,1$ and $m=0,1, \ldots, \mu_{a, y}(C)$ is the probability measure of the distance between $\tau_{2 m+a+1}$ and $\tau_{2 m+a}$, dependent on $a$ and on the condition $\left\{X\left(\tau_{2 m+a}\right)=y\right\}$.

The moments $\tau_{2 m+a}, a=0,1, m=0,1, \ldots$, are called regenerative moments of the process and the intervals $\tau_{m} \leqslant t<\tau_{m+1}$ are called Markovian intervals. Changes of the state of the process in the interval $\tau_{m}<t$ $<\tau_{m+1}$ are called Markovian transitions and changes of the state at the regenerative moments are called regenerative transitions.

Let $X_{m}^{-}, m=1,2, \ldots$, denote the state of the process $\{X(t), t \geqslant 0\}$ at the moment $\tau_{m}$ just before the last regenerative transition. Consider four sequences of random variables:

$$
\left\{X_{2 m-a}^{-}, m=1,2, \ldots\right\}, \quad\left\{X\left(\tau_{2 m+a}\right), m=0,1, \ldots\right\}, \quad a=0,1 .
$$

It is easy to see that they form Markov chains, since $\tau_{2 m+a}, a=0,1$, $m=0,1, \ldots$, are regenerative moments of the process $\{X(t), t \geqslant 0\}$.

Assume that there exists a probability measure $N_{0}^{-}$being invariant for the chain $\left\{X_{2 m}^{-}, m=1,2, \ldots\right\}$. Then it is easy to find invariant measures $N_{1}^{-}, N_{0}^{+}, N_{1}^{+}$for the chains

$$
\begin{aligned}
\left\{X_{2 m-1}^{-}, m=1,2, \ldots\right\}, \quad\left\{X\left(\tau_{2 m}\right), m=0,1, \ldots\right\}, & \left\{X\left(\tau_{2 m+1}\right), m=0,1, \ldots\right\},
\end{aligned}
$$

respectively.

In the sequel of this paper we find relations between the stationary probability distribution of the process $\{X(t), t \geqslant 0\}$ and the probability measures $N_{a}^{-}, N_{a}^{+}, a=0,1$. Sufficient conditions for the ergodicity of Markov chains on a general state space are given in [13]. 


\section{EXTENDED MARKOV PROCESS}

Let $\{X(t), t \geqslant 0\}$ be an extended piecewise Markov process with regenerative moments $\left\{\tau_{m}, m=0,1, \ldots\right\}$. Assume that

$$
\sup _{m \geqslant 0} \tau_{m}=+\infty \quad \text { Pr-almost everywhere }
$$

and define the following processes related to the process $\{X(t), t \geqslant 0\}$ :

$$
\begin{aligned}
& Y(t)=X\left(\tau_{2 m+a}\right), \\
& Z(t)=\tau_{2 m+a+1}-t, \\
& \alpha(t)=a,
\end{aligned} \quad \tau_{2 m+a} \leqslant t<\tau_{2 m+a+1}, a=0,1, m=0,1, \ldots
$$

The process $\{Y(t), t \geqslant 0\}$ is called a semi-Markov process, $\{Z(t), t \geqslant 0\}$ a residual-time process, and $\{\alpha(t), t \geqslant 0\}$ a breakdown process.

Let

$$
\begin{gathered}
\overline{\mathscr{X}}=\mathscr{X}^{2} \times \mathscr{R}_{+} \times\{0,1\}, \quad \bar{x}=(x, y, z, i), \quad \check{x}=(x, x, z, i), \\
\bar{x}_{1}=\left(x_{1}, y_{1}, z_{1}, j\right), \quad \check{x}_{1}=\left(x_{1}, x_{1}, z_{1}, j\right), \quad \bar{x}_{2}=\left(x_{2}, y_{2}, z_{2}, a\right), \\
\bar{x}, \check{x}_{1}, \bar{x}_{1}, \check{x}_{1}, \bar{x}_{2} \in \bar{X}, \quad \tilde{x}=(x, y, z) \in \mathscr{X}^{2} \times \mathscr{R}_{+}, \\
\overline{\mathscr{F}}=\mathscr{F}^{2} \times \mathscr{B}_{+} \times \mathscr{B}_{1}, \quad \bar{A}=A \times B \times C \times\{a\} \in \overline{\mathscr{F}} \\
\bar{X}(t)=(\bar{X}(t), \bar{Y}(t), Z(t), a(t)), \\
\bar{P}(t, \bar{x}, \bar{A})=\operatorname{Pr}(\bar{X}(t) \in \bar{A} \mid \bar{X}(0)=\bar{x}), \quad t \geqslant 0 .
\end{gathered}
$$

Let us define the transition probabilities

$$
\bar{\Pi}(t, \bar{x}, \bar{A})=P_{i, y}(t, x, A) I_{B}(y) I_{C+t}(z) \delta_{i, a}, \quad t \geqslant 0,
$$

where $\delta$ is Kronecker's delta, $C+t=\{c+t: c \in C\}, I_{A}$ is the indicator of the set $A$,

(2) $S_{1}\left(\bar{x}, A \times C_{1} \times\{j\}\right)=\delta_{1-i, j} \int_{\mathscr{X}} P_{i, y}\left(z, x, d s_{1}\right) \int_{A} Q_{i}\left(s_{1}, d x_{1}\right) \mu_{j, x_{1}}\left(C_{1}\right)$,

(3) $\quad S_{k}\left(\bar{x}, A \times C_{1} \times C_{2} \times \ldots \times C_{k} \times\{j\}\right)=\delta_{e(k, i), j} \int_{x} P_{i, y}\left(z, x, d s_{1}\right) \times$

$\times \int_{\mathscr{U}} Q_{i}\left(s_{1}, d x_{1}\right) \int_{C_{1}} \mu_{1-i, x_{1}}\left(d z_{1}\right) \prod_{r=1}^{k-2} \int_{\mathscr{X}} P_{\mathrm{e}(r, i), x_{r}}\left(z_{r}, x_{r}, d s_{r+1}\right) \times$

$\times \int_{\mathscr{X}} Q_{\Theta(r, i)}\left(s_{r+1}, d x_{r+1}\right) \int_{C_{r+1}} \mu_{\varrho(r+1, i) j, x_{r+1}}\left(d z_{r+1}\right) \int_{\mathscr{X}} P_{1-j, x_{k-1}}\left(z_{k-1}, x_{k-1}, d s_{k}\right) \times$ $\times \int_{A} Q_{1-j}\left(s_{k}, d x_{k}\right) \mu_{j, x_{k}}\left(C_{k}\right), \quad i, j=0,1, k=2,3, \ldots$,

$A \in \mathscr{F}, C_{l} \in \mathscr{B}_{0}$ for $l=1,2, \ldots, k-1, C_{k} \in \mathscr{B}_{+}$, and $\varrho(r, i)$ equals $i$ for even $r$ and equals $1-i$ for odd $r$. 
THEOREM 1. The process $\{\bar{X}(t), t \geqslant 0\}$ is a homogeneous Markov process with transition probabilities $\bar{P}(t, \bar{x}, \bar{A})$ satisfying

$$
\begin{aligned}
\bar{P}(t, \bar{x}, \bar{A})= & \bar{\Pi}(t, \bar{x}, \bar{A})+I_{(0, t]}(z) \int_{\mathscr{X} \times \mathscr{R}_{+}}\left[S_{1}\left(\bar{x}, d x_{1} \times d z_{1} \times\{j\}\right)+\right. \\
& \left.+S_{2}\left(\bar{x}, d x_{1} \times\{0\} \times d z_{1} \times\{j\}\right)\right] \bar{P}\left(t-z, \check{x}_{1}, \bar{A}\right),
\end{aligned}
$$

where $\bar{\Pi}(t, \bar{x}, \bar{A})$ are transition probabilities defined by (1). The explioit form of the probabilities $\bar{P}(t, \bar{x}, \bar{A})$ may be expressed by the formula

$$
\begin{aligned}
& \bar{P}(t, \bar{x}, \bar{A})=\bar{\Pi}(t, \bar{x}, \bar{A})+ \\
+ & \sum_{k=1}^{\infty} \sum_{j=0}^{1} \int_{\mathscr{X} \times \mathscr{R}_{0}^{k-1} \times \mathscr{R}_{+}} s_{k}\left(\dot{\bar{x}}, d x_{1} \times d z_{1} \times \ldots \times d z_{k} \times\{j\}\right) \times \\
\times & I_{(0, t]}\left(z+z_{1}+\ldots+z_{k-1}\right) \bar{\Pi}\left(t-z-z_{1}-\ldots-z_{k-1},\left(x_{1}, x_{1}, z_{k}, j\right), \bar{A}\right), \\
& t \geqslant 0, \bar{x} \in \overline{\mathscr{X}}, \bar{A} \in \overline{\mathscr{F}},
\end{aligned}
$$

where $S_{k}, k \geqslant 1$, are transition probabilities defined by (2) and (3).

Proof. We prove formula (5). Let, for $s, t>0$,

$$
r_{1}(s)=\max _{m \geqslant 1}\left\{m: \tau_{m} \leqslant s\right\}, \quad r(s, s+t)=r_{1}(s+t)-r_{1}(s) .
$$

Hence we have

$$
\bar{P}(t, \bar{x}, \bar{A})=\sum_{k=0}^{\infty} \operatorname{Pr}(\bar{X}(s+t) \in \bar{A} \cap r(s, s+t)=k \mid \bar{X}(s)=\bar{x})
$$

If $r(s, s+t)=0$, then in the interval $(s, s+t]$ there are no regenerative moments. Therefore, we have

$$
\operatorname{Pr}(\bar{X}(s+t) \in \bar{A} \cap r(s, s+t)=0 \mid \bar{X}(s)=\bar{x})=\bar{\Pi}(t, \bar{x}, \bar{A}) .
$$

If $r(s, s+t)=k \geqslant 1$, then in the interval $(s, s+t]$ there are exactly $k$ regenerative moments

$$
\ldots s<\tau_{l} \leqslant \tau_{l+1} \leqslant \ldots \leqslant \tau_{l+k-1} \leqslant s+t<\tau_{l+k} \ldots
$$

Hence we have

$$
\begin{aligned}
& \operatorname{Pr}(\bar{X}(s+t) \in \bar{A} \cap r(s, s+t)=k \mid \bar{X}(s)=\bar{x}) \\
= & \sum_{j=0}^{1} \int_{\mathscr{X} \times \mathscr{R}_{0}^{k-1} \times \mathscr{R}_{+}} S_{k}\left(\bar{x}, d x_{1} \times d z_{1} \times \ldots \times d z_{k} \times\{j\}\right) I_{(0, t]}\left(z+z_{1}+\ldots+z_{k-1}\right) \times \\
& \times \bar{\Pi}\left(t-z-z_{1}-\ldots-z_{k-1},\left(x_{1}, x_{1}, z_{k}, j\right), \bar{A}\right),
\end{aligned}
$$


where

$$
\begin{aligned}
\int_{\mathscr{R}_{0}^{k-1}} S_{k}\left(\bar{x}, A \cap B \times d z_{1}\right. & \left.\times \ldots \times d z_{k-1} \times C_{k} \times\{j\}\right) \\
& =\operatorname{Pr}\left(\bar{X}\left(\tau_{l+k-1}\right) \in A \times B \times C_{k} \times\{j\} \mid \bar{X}(s)=\bar{x}\right) .
\end{aligned}
$$

Formula (4) can be obtained analogously.

The transition probabilities $\bar{P}$ and $\bar{\Pi}$ defined on the space $(\overline{\mathscr{X}}, \bar{F})$ induce two semi-groups of contraction operators $\left\{\overline{\mathscr{P}}_{t}, t \geqslant 0\right\}$ and $\left\{\bar{\pi}_{t}, t \geqslant 0\right\}$ defined by the formulas

$$
\begin{array}{r}
\overline{\mathscr{P}}_{t} \bar{M}(\bar{A})=\int_{\bar{X}} \bar{M}(d \bar{x}) \bar{P}(t, \bar{x}, \bar{A}), \quad \bar{\pi}_{t} \bar{M}(\bar{A})=\int_{\bar{x}} \bar{M}(d \bar{x}) \bar{\Pi}(t, \bar{x}, \bar{A}), \\
t \geqslant \mathbf{0}, \bar{A} \in \overline{\mathscr{F}},
\end{array}
$$

where $\bar{M}$ is an arbitrary probability measure on the space $(\overline{\mathscr{X}}, \overline{\mathscr{F}})$ $(\bar{M} \in \overline{\mathscr{M}}(\overline{\mathscr{X}}, \overline{\mathscr{F}})=\overline{\mathscr{M}})$.

In the sequel we apply theorems from contraction-semi-group theory included in [3], p. 59-65. To do this we introduce $\omega$-weak convergence in the set of probability measures $\overline{\mathscr{M}}$. Denote by $\mathscr{C}_{0}(\mathscr{X})$ the set of all realvalued continuous functions vanishing at infinity,

$$
\lim _{x \rightarrow \pm \infty} f(x)=\mathbf{0}, \quad f \in \mathscr{C}_{\mathbf{0}}(\mathscr{X}),
$$

defined on $\mathscr{X}$. Let $\overline{\mathscr{C}}_{0}=\mathscr{C}_{0}\left(\mathscr{X}^{2} \times \mathscr{R}_{+}\right)$. For the conjugate space $\overline{\mathscr{C}}_{0}^{*}$ by the Riesz theorem (see [12], p. 312) we have $\overline{\mathscr{C}}_{0}^{*} \supset \overline{\mathscr{M}}$. Thus we may define the $\omega$-weak convergence as follows:

Definition 2. The sequence of measures $\bar{M}_{n} \in \overline{\mathscr{M}}, n=1,2, \ldots$, is w-weakly convergent to the measure $\bar{M} \in \overline{\mathscr{M}}$,

if for any arbitrary function $f \in \overline{\mathscr{C}}_{0}$ and for every $i=0,1$ we have

$$
\begin{aligned}
& \omega-\lim _{n \rightarrow \infty} \bar{M}_{n}(\bar{A})=\bar{M}(\bar{A}), \quad \bar{A} \in \mathscr{F}, \\
& \text { function } f \in \overline{\mathscr{C}}_{0} \text { and for every } i=0,1 \\
& \int_{\mathscr{X}^{2} \times \mathscr{R}_{+}} \bar{M}_{n}(d \bar{x}) f(\tilde{x})=\int_{\mathscr{X}^{2} \times \mathscr{R}_{+}} \bar{M}(d \bar{x}) f(\tilde{x}) .
\end{aligned}
$$

For semi-groups of contraction operators $\left\{\overline{\mathscr{P}}_{t}, t \geqslant 0\right\}$ and $\left\{\bar{\pi}_{t}, t \geqslant 0\right\}$ denote by $\mathscr{A}(\bar{P})$ and $\mathscr{A}(\bar{\Pi})$ the infinitesimal operators, by $\mathscr{R}_{\lambda}(\bar{P})$ and $\mathscr{R}_{\lambda}(\bar{\Pi}), \lambda>0$, the resolvents, and by $\mathscr{R}(\bar{P})$ and $\mathscr{R}(\bar{\Pi})$ the potentials, in the sense of $\omega$-weak convergence (see $[3]$, p. 59-65). Let us introduce the following notation for the kernels of resolvents:

$$
\begin{aligned}
\bar{P}_{\lambda}(\bar{x}, \bar{A})=\int_{\mathscr{R}_{+}} e^{-\lambda t} \bar{P}(t, \bar{x}, \bar{A}) d t, \quad \bar{\Pi}_{\lambda}(\bar{x}, \bar{A}) & =\int_{\mathscr{R}_{+}} e^{-\lambda t} \bar{\Pi}(t, \bar{x}, \bar{A}) d t, \\
\lambda & >0, \quad \bar{x} \in \overline{\mathscr{X}}, \bar{A} \in \overline{\mathscr{F}} .
\end{aligned}
$$


Multiplying (5) by $e^{-\lambda t}$ and integrating over $\mathscr{R}_{+}$we obtain the following corollary:

CoRoLlary 1 . The kernels of resolvents $\bar{P}_{\lambda}(\bar{x}, \bar{A})$ and $\bar{\Pi}_{\lambda}(\bar{x}, \bar{A})$ satisfy the equality

$$
\bar{P}_{\lambda}(\bar{x}, \bar{A})=\bar{\Pi}_{\lambda}(\bar{x}, \bar{A})+\int_{\overline{\mathscr{X}}} S_{\lambda}(\bar{x}, d \bar{y}) \bar{\Pi}_{\lambda}(\bar{y}, \bar{A}), \quad \lambda>0, \bar{x}, \bar{y} \in \overline{\mathscr{X}}, \bar{A} \in \overline{\mathscr{F}},
$$

where

$$
\begin{aligned}
S_{\lambda}(\bar{x}, \bar{A})= & \sum_{k=1}^{\infty} \int_{\mathscr{R}_{0}^{k-1}} \exp \left[-\lambda\left(z+z_{1}+z_{2}+\ldots+z_{k-1}\right)\right] \times \\
& \times S_{k}\left(\bar{x}, A \cap B \times d z_{1} \times \ldots \times d z_{k-1} \times C \times\{a\}\right), \quad \bar{x} \in \overline{\mathscr{X}}, \bar{A} \in \overline{\mathscr{F}} .
\end{aligned}
$$

\section{STATIONARY EXTENDED MARKOV PROCESS}

We investigate now the stationary Markov process

$$
\{\bar{X}(t), t \geqslant 0\}=\{(X(t), Y(t), Z(t), \alpha(t)), t \geqslant 0\}
$$

defined in section 2 (for the definition of the stationary stochastic process see $[4]$, p. 165). The stationary probability distribution $\bar{N}(\bar{A})=\operatorname{Pr}(\bar{X}(t) \in \bar{A})$ is an invariant measure for the semi-group of operators $\left\{\overline{\mathscr{P}}_{t}, t \geqslant 0\right\}$, i.e. $\overline{\mathscr{P}}_{t} \bar{N}(\bar{A})=\bar{N}(\bar{A}), \bar{A} \in \overline{\mathscr{F}}$, for every $t \geqslant 0$. We assume that there exists a unique invariant measure for the semi-group $\left\{\overline{\mathscr{P}}_{t}, t \geqslant 0\right\}$.

Let us introduce the following conditions (i)-(iv):

(i) For $a=0,1$ there exist limits

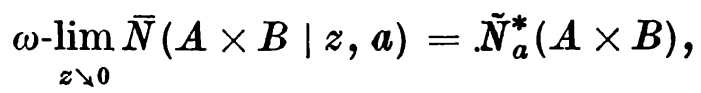

where

$$
\bar{N}(A \times B \mid z, a)=\operatorname{Pr}(X(t) \in A, Y(t) \in B \mid Z(t) \leqslant z, \alpha(t)=a) .
$$

(ii) For every $f \in \mathscr{C}_{0}\left(\mathscr{X}^{2}\right), g \in \mathscr{C}_{0}\left(\mathscr{R}_{+}\right)$, and $a=0,1$ we have

$$
\begin{gathered}
\lim _{t \searrow 0} \sup _{x, y \in \mathscr{X}}\left|\int_{\mathscr{X}} P_{a, y}\left(t, x, d x_{1}\right) f\left(x_{1}, y\right)-f(x, y)\right|=0, \\
\quad \underset{t \rtimes 0}{\lim \sup _{x \in \mathscr{X}}}\left|\int_{\mathscr{R}_{+}} \mu_{a, x}(d z)(g(z-t)-g(z))\right|=0 .
\end{gathered}
$$


(iii) For every $f \in \overline{\mathscr{C}}_{0}, a=0,1$, and $x \in \mathscr{X}$ the functions

$$
\begin{aligned}
w_{a}(x) & =\int_{\mathscr{X}} Q_{a}(x, d y) \int_{\mathscr{R}_{+}} \mu_{a, y}(d z) f(y, y, z) \\
w(x) & =\int_{\mathscr{X}} Q_{1}(x, d y) \mu_{0, y}(\{0\}) \int_{\mathscr{X}} Q_{0}(y, d s) \int_{\mathscr{R}_{+}} \mu_{1, s}(d z) f(s, s, z)
\end{aligned}
$$

belong to $\mathscr{C}_{0}(\mathscr{X})$. that

(iv) There exist functions $\varepsilon_{a}(t), t \geqslant 0, \lim _{t \searrow 0} \varepsilon_{a}(t)=0, a=0,1$, such

$$
\sup _{x \in \mathscr{X}} \mu_{a, x}((0, t]) \leqslant \varepsilon_{a}(t)
$$

Under these conditions we prove the following theorem:

THEOREM 2. If $\vec{N}$ is a unique invariant probability measure for the semi-group of operators $\left\{\overline{\mathscr{P}}_{t}, t \geqslant 0\right\}$ and if conditions (i)-(iv) are fulfilled,
then

(a) the measure $\bar{N}$ satisfies the equations

where

$$
\mathscr{A}(\bar{\Pi}) \bar{N}(\bar{A})+v \bar{N}_{a}^{+}(A \times B \times C)=0, \quad a=0,1,
$$

$$
\begin{gathered}
\bar{N}_{a}^{+}(A \times B \times C)=\int_{A} N_{a}^{+}(d x) I_{B}(x) \mu_{a, x}(C), \quad \frac{1}{v}=\sum_{a=0}^{1} \int_{\mathscr{X}} N_{a}^{+}(d s) m_{a, 8}, \\
m_{a, y}=\int_{\mathscr{R}_{+}} t \mu_{a, y}(d t), \quad y \in \mathscr{X}, \quad A, B \in \mathscr{F}, \quad C \in \mathscr{B}_{+},
\end{gathered}
$$

provided $v<\infty$;

(b) the marginal measurès $N_{a}(A)=\bar{N}\left(A \times \mathscr{X} \times \mathscr{R}_{+} \times\{a\}\right)$ and the measures $N_{a}^{+}$fulfil the relations

(8)

$$
N_{a}(A)=v \int_{\mathscr{X}} N_{a}^{+}(d x) \int_{\mathscr{R}_{+}} P_{a, x}(t, x, A) \mu_{a, x}((t, \infty)) d t, \quad a=0,1, A \in \mathscr{F}
$$

provided the right-hand side of (8) is finite;

(c) the marginal measures $v_{a}(C)=\bar{N}\left(\mathscr{X}^{2} \times C \times\{a\}\right)$ are absolutely continuous and we have

(9)

$$
v_{a}(C)=v \int_{C} h_{a}(z) d z, \quad a=0,1, \quad \text { where } \quad h_{a}(z)=\int_{\mathscr{X}} N_{a}^{+}(d x) \mu_{a, x}((z, \infty))
$$

Before we start the proof of theorem 2, we prove four lemmas. 

we have

LEMMA 1. If condition (ii) is fulfilled, then for every measure $\bar{M} \in \overline{\mathscr{M}}$

$$
\omega-\lim _{t>0} \bar{\pi}_{t} \bar{M}(\bar{A})=\bar{M}(\bar{A}), \quad \bar{A} \in \overline{\mathscr{F}} \text { or } \bar{M} \in \mathscr{L}(\bar{\Pi}) .
$$

Proof. It suffices to show that for arbitrary $\bar{x} \in \bar{X}$ and $a=0,1$

$$
\omega-\lim _{t \searrow 0} \bar{\Pi}(t, \bar{x}, \cdot)=I_{(\cdot)}(\bar{x}) .
$$

Then the lemma follows frorn Lebesgue's theorem on bounded convergence.

It follows from (1) that the extended measure $\bar{\Pi}(t, \bar{x}, \cdot)$ is a product measure. In that case, $\omega$-weak convergence $\bar{\Pi}(t, \bar{x}, \cdot)$ is equivalent to the $\omega$-weak convergence of measures $P_{i, y}(t, x, \cdot) I_{(\cdot)}(y)$ and $I_{(\cdot)+t}(z)$ (see [2], p 21). Hence it suffices to show that for every function $f \in \mathscr{C}_{0}\left(\mathscr{X}^{2}\right)$ we have

$$
\lim _{t \searrow 0} \int_{\mathscr{X}} P_{a, y}(t, x, d u) \int_{\mathscr{X}} I_{d s}(y) f(u, s)=\lim _{t \succ 0} P_{a, y}(t, x, d u) f(u, y)=f(x, y) .
$$

This fact follows immediately from (ii).

LEMMA 2. If condition (ii) is fulfilled and if for every measure $\bar{M} \in \bar{M}$ there exists

$$
\omega-\lim _{t>0} \frac{\overline{\mathscr{P}}_{t} \bar{M}(\bar{A})-\bar{M}(\bar{A})}{t}, \quad \bar{A} \in \overline{\mathscr{F}} \text { or, equivalently, } \bar{M} \in \mathscr{D}(\bar{P}),
$$

then there exists

(11) $\quad \omega-\lim _{t>0} \frac{\bar{\pi}_{t} \bar{M}(\bar{A})-\bar{M}(\bar{A})}{t}, \quad \bar{A} \in \overline{\mathscr{F}}$ or, equivalently, $\bar{M} \in \mathscr{D}(\bar{\Pi})$.

Proof. By the resolvent theorem ([3], p. 65, theorem 1,7), if $\bar{M} \in \mathscr{D}(\bar{P})$ then there exists the measure

$$
\bar{M}_{1}=\lambda \bar{M}-\mathscr{A}(\bar{P}) \bar{M} \in \mathscr{L}(\bar{P})
$$

such that $\bar{M}=\mathscr{R}_{\lambda}(\bar{P}) \bar{M}_{1}, \lambda>0$. Hence by (6) we have

$$
\begin{aligned}
\mathscr{R}_{\lambda}(\bar{P}) \bar{M}_{1}(\bar{A}) & =\int_{\overline{\mathscr{X}}} \bar{M}_{1}(d \bar{x}) \bar{P}_{\lambda}(\bar{x}, \bar{A}) \\
& =\mathscr{R}_{\lambda}(\bar{\Pi}) \bar{M}_{1}(\bar{A})+\int_{\overline{\mathscr{X}}}\left(\int_{\overline{\mathscr{X}}} \bar{M}_{1}(d \bar{x}) S_{\lambda}(\bar{x}, d \bar{y})\right) \bar{\Pi}_{\lambda}(\bar{y}, \bar{A}) .
\end{aligned}
$$

Since the resolvent $\mathscr{R}_{\lambda}(\bar{\Pi})$ is a one-to-one mapping of $\mathscr{L}(\bar{\Pi})$ on $\mathscr{D}(\bar{\Pi})$, it suffices to show that the measures $\bar{M}_{1}$ and

$$
\bar{M}_{1, \lambda}(\bar{A})=\int_{\overline{\mathscr{X}}} \bar{M}_{1}(d \bar{x}) S_{\lambda}(\bar{x}, \bar{A}), \quad \lambda>0, \bar{A} \in \overline{\mathscr{F}},
$$

belong to $\mathscr{L}(\bar{\Pi})$. 
This fact follows from (10).

LEMMA 3. If condition (ii) is fulfilled, then for every $f \in \overline{\mathscr{C}}_{0}$ and $i, a=0,1$ we have

$$
\begin{aligned}
\limsup & \int_{t \backslash 0} \mu_{i, x}(d z) \int_{\mathscr{X}^{2} \times \mathscr{R}_{+}} \bar{\Pi}\left(t, \check{x}, d \bar{x}_{1}\right) f\left(\tilde{x}_{1}\right)- \\
& \quad-\delta_{i, a} \int_{\mathscr{R}_{+}} \mu_{i, x}(d z) f(x, x, z) \mid=0 .
\end{aligned}
$$

Proof. Let $f(\tilde{x})=g(x, y) h(z), g \in \mathscr{C}_{0}\left(\mathscr{X}^{2}\right), h \in \mathscr{C}_{\eta}\left(\mathscr{R}_{+}\right)$. By (ii) for every $\varepsilon>0$ and sufficiently small $t$ we have

$$
\begin{aligned}
& \sup _{x \in \mathscr{X}} \mid \int_{\mathscr{R}_{+}} \mu_{i, x}(d z) \int_{\mathscr{X}^{2} \times \mathscr{R}_{+}} \bar{\Pi}\left(t, \check{x}, d \bar{x}_{1}\right) g\left(x_{1}, y_{1}\right) h\left(z_{1}\right)- \\
& \quad-\delta_{i, a} g(x, x) \int_{\mathscr{R}_{+}} \mu_{i, x}(d u) h(u) \mid \\
& =\delta_{i, a} \sup _{x \in \mathscr{X}}\left|\int_{\mathscr{R}_{+}} \mu_{i, x}(d z) h(z-t) \int_{\mathscr{X}} P_{i, x}(t, x, d u) g(u, x)-g(x, x) \int_{\mathscr{R}_{+}} \mu_{i, x}(d z) h(z)\right| \\
& \leqslant \varepsilon \sup _{t \in \mathscr{R}_{+}}|h(t)|+\varepsilon \sup _{(x, y) \in \mathscr{T}^{2}}|g(x, y)| .
\end{aligned}
$$

Since $\overline{\mathscr{C}}_{0}$ is generated by functions of the form $f(\tilde{x})=g(x, y) h(z)$ (see [12], p. 117), the proof is completed.

LEMCMA 4. If condition (ii) is fulfilled, then there exists $c>0$ such that

$$
v((0, t])=\sum_{a=0}^{1} \bar{N}\left(\mathscr{X}^{2} \times(0, t] \times\{a\}\right) \leqslant c t, \quad t>0 .
$$

Proof. By (11) we have $\bar{N} \in \mathscr{D}(\bar{\Pi})$. Hence, by the resolvent theorem (see [3], p. 65), there exists a measure $\bar{M}=\lambda \bar{N}-\mathscr{A}(\bar{\Pi}) \bar{N}$ such that $\bar{N}=\mathscr{R}_{\lambda}(\bar{\Pi}) \bar{M}, \lambda>0$. Thus we have

where

$$
v(C)=\int_{\mathscr{R}_{+}} e^{-\lambda t} \int_{\mathscr{R}_{+}} \varphi(d z) I_{C+t}(z) d t
$$

$$
\varphi(C)=\sum_{i=0}^{1} \bar{M}\left(\mathscr{X}^{2} \times C \times\{i\}\right), \quad C \in \mathscr{B}_{+} .
$$

In particular, we have

$$
v((0, t]) \leqslant \varphi^{+}\left(\mathscr{R}_{+}\right) t, \quad \text { where } \varphi^{+}=\max (0, \varphi) .
$$
we have

Proof of theorem 2. For the invariant probability measure $\bar{N}$

$$
\mathscr{A}(\bar{P}) \bar{N}(\bar{A})=\omega-\lim _{t>0} \frac{\overline{\mathscr{P}}_{t} \bar{N}(\bar{A})-\bar{N}(\bar{A})}{t}=0, \quad \bar{A} \in \overline{\mathscr{F}}
$$


Hence, using formula (4), we obtain

$$
\begin{aligned}
\omega-\lim _{t \rtimes 0} \frac{\bar{\pi}_{t}}{\bar{N}(\bar{A})-\bar{N}(\bar{A})} & t \\
& +\omega-\lim _{t \rtimes 0} \frac{1}{t} \int_{\bar{x}} \bar{N}(d \bar{x}) I_{(0, t]}(z) \int_{\mathscr{X} \times \mathscr{R}_{+}}\left[S_{1}\left(\bar{x}, d x_{1} \times d z_{1} \times\{j\}\right)+\right. \\
& \left.+S_{2}\left(\vec{x}, d x_{1} \times\{0\} \times d z_{1} \times\{j\}\right)\right] \bar{P}\left(t-z, \check{x}_{1}, \bar{A}\right)=0 .
\end{aligned}
$$

It follows from lemma 2 that $\bar{N} \in \mathscr{D}(\bar{\Pi})$, therefore the first expression on the left-hand side of (14) exists and equals $\mathscr{A}(\bar{\Pi}) \bar{N}(\bar{A}), \bar{A} \in \overline{\mathscr{F}}$.

Now we show that in (14) we may replace the expression $\bar{P}\left(t-z, \check{x}_{1}, \bar{A}\right)$ by the expression $\bar{\Pi}\left(t-z, \check{x}_{1}, \bar{A}\right)$. Indeed, for every function $f \in \overline{\mathscr{C}}_{0}$, by (4), (iv), and (13), we have

$$
\begin{aligned}
& \frac{1}{t} \int_{\bar{x}} \bar{N}(d \bar{x}) I_{(0, t]}(z) \int_{\mathscr{X} \times \mathscr{T}_{+}}\left[S_{1}\left(\bar{x}, d x_{1} \times d z_{1} \times\{j\}\right)+S_{2}\left(\bar{x}, d x_{1} \times\{0\} \times\right.\right. \\
& \left.\left.\quad \times d z_{1} \times\{j\}\right)\right] \times \int_{x^{2} \times \mathscr{R}_{+}}\left[\bar{P}\left(t-z, \check{x}_{1}, d \bar{x}_{2}\right)-\bar{\Pi}\left(t-z, \check{x}_{1}, d \bar{x}_{2}\right)\right] f\left(\tilde{x}_{2}\right) \\
& \leqslant \sup _{\tilde{x}_{2} \in \mathscr{X}^{2} \times \mathscr{R}_{+}}\left|f\left(\tilde{x}_{2}\right)\right| \frac{1}{t} \int_{\overline{\mathscr{X}}} \bar{N}(d \bar{x}) I_{(0, t]}(z) \int_{x^{2} \times \mathscr{T}_{+}}\left[S_{1}\left(\bar{x}, d x_{1} \times(0, t-z] \times\{j\}\right)+\right. \\
& \left.+S_{2}\left(\bar{x}, d x_{1} \times\{0\} \times(0, t-z] \times\{j\}\right)\right] \\
& \leqslant \sup _{\tilde{x}_{2} \in \mathscr{X}^{2} \times \mathscr{R}_{+}}\left|f\left(\tilde{x}_{2}\right)\right| 2 c \varepsilon(t) \rightarrow 0 \quad \text { for } t \searrow 0,
\end{aligned}
$$

where

$$
\varepsilon(t)=\max _{i=0,1} \varepsilon_{i}(t), \quad t \geqslant 0 .
$$

Using (12) and (13) for every $\varepsilon>0$ and sufficiently small $t$ we have

$$
\begin{aligned}
\frac{1}{t} \int_{\overline{\mathscr{X}}} \bar{N}(d \bar{x}) I_{(0, t]}(z) & \int_{\mathscr{X}} P_{i, y}(z, x, d s) \int_{\mathscr{X}} Q_{i}\left(s, d x_{1}\right) \sup _{x_{1} \in \mathscr{C}} \mid \int_{\mathscr{R}_{+}} \mu_{1-i, x_{1}}\left(d z_{1}\right) \times \\
& \times \int_{\mathscr{X}^{2} \times \mathscr{R}_{+}} \bar{\Pi}\left(t-z,\left(x_{1}, x_{1}, z_{1}, 1-i\right), d \bar{x}_{2}\right) f\left(\tilde{x}_{2}\right)- \\
& -\delta_{1-i, a} \int_{\mathscr{C}_{+}} \mu_{1-i, x_{1}}\left(d z_{1}\right) f\left(x_{1}, x_{1}, z_{1}\right) \mid \leqslant c \varepsilon .
\end{aligned}
$$


From (iii) and (ii) we obtain

$$
\begin{aligned}
& \text { 16) } \frac{1}{t} \sum_{i=0}^{1} \int_{0}^{t} \int_{\mathscr{X}^{2}} \bar{N}(\bar{d} \bar{x}) \delta_{1-i, a} \sup _{x, y \in \mathscr{X}} \mid \int_{\mathscr{X}} P_{i, y}(z, x, d s) \int_{\mathscr{X}} Q_{i}\left(s, d x_{1}\right) \times \\
& \times \int_{\mathscr{R}_{+}} \mu_{1-i, x_{1}}\left(d z_{1}\right) f\left(x_{1}, x_{1}, z_{1}\right)-\int_{\mathscr{X}} Q_{i}\left(x, d x_{1}\right) \int_{\mathscr{R}_{+}} \mu_{1-i, x_{1}}\left(d z_{1}\right) f\left(x_{1}, x_{1}, z_{1}\right) \mid \leqslant 2 c \varepsilon .
\end{aligned}
$$

Let us assume for the moment that

$$
v_{a}(C)=\int_{C} h_{a}(z) d z \quad \text { and } \quad \lim _{z \searrow 0} h_{a}(z)=v_{a}, \quad a=0,1
$$

Then using (15), (16), (17), (i), and (iii) we obtain

$$
\begin{aligned}
\lim _{t>0} \frac{1}{t} \sum_{i=0}^{1} \int_{0}^{t} \int_{\mathscr{X}^{2}} \bar{N}(d \bar{x}) \int_{\mathscr{X}} P_{i, y}\left(z, x, d s_{1}\right) \int_{\mathscr{X}} Q_{i}\left(s_{1}, d x_{1}\right) \int_{\mathscr{R}_{+}} \mu_{1-i, x_{1}}\left(d z_{1}\right) \times \\
\quad \times \int_{\mathscr{X}^{2} \times \mathscr{R}_{+}} \bar{\Pi}\left(t-z,\left(x_{1}, x_{1}, z_{1}, 1-i\right), d \bar{x}_{2}\right) f\left(\tilde{x}_{2}\right) \\
=v_{1-a} \int_{\mathscr{X}^{2}} \tilde{N}_{1-a}^{*}(d x \times d y) \int_{\mathscr{X}} Q_{1-a}\left(x, d x_{1}\right) \int_{\mathscr{x}_{+}} \mu_{a, x_{1}}\left(d z_{1}\right) f\left(x_{1}, x_{1}, z_{1}\right) .
\end{aligned}
$$

Analogously, we may compute the last limit of (14).

Hence using the notation $N_{a}^{*}(A)=\tilde{N}_{a}^{*}(A \times \bar{X}), a=0,1$, we obtain the following form of equation (14):

$$
\begin{gathered}
\mathscr{A}(\bar{\Pi}) \bar{N}(\bar{A})+v_{1-a} \int_{\mathscr{X}} N_{1-a}^{*}(d s) \int_{\dot{A}} Q_{1-a}(s, d x) I_{B}(x) \mu_{a, x}(C)+ \\
+v_{a} \int_{\mathscr{X}} N_{a}^{*}(d s) \int_{\mathscr{X}} Q_{a}(s, d u) \mu_{1-a, u}(\{0\}) \int_{\mathcal{A}} Q_{1-a}(u, d x) I_{B}(x) \mu_{a, x}(C)=0 .
\end{gathered}
$$

If the measure $\bar{M} \in \overline{\mathscr{M}}$ fulfils the equation $\mathscr{A}(\bar{P}) \bar{M}=0$, then by the resolvent theorem there exists the probability measure $\bar{M}_{1} \in \mathscr{L}(\bar{P})$ such
that

$$
\bar{M}=\mathscr{R}_{1}(\bar{P}) \bar{M}_{1} \quad \text { and } \quad \bar{M}_{1}=\bar{M}-\mathscr{A}(\bar{P}) \bar{M}
$$

Then we have $\bar{M}=\mathscr{R}_{1}(\bar{P}) \bar{M}$ and it suffices for the measure to be invariant for the semi-group $\left\{\overline{\mathscr{P}}_{t}, t \geqslant 0\right\}$ (see [1]). By the assumption, there exists a unique invariant measure $\bar{N}$, thus the equation $\mathscr{A}(\bar{P}) \bar{M}=0$ has a unique solution. We show that every solution of equation (18) fulfils assumption (17); therefore, this assumption is always fulfilled. 
By the potential theorem (see [3], p. 65, theorem 1,7'), we have

$$
\begin{aligned}
& v_{i}((\tau, \infty))=v_{1-i} \int_{\mathscr{X}} N_{1-i}^{*}(d s) \int_{\mathscr{X}} Q_{1-i}(s, d x) \int_{\mathscr{R}_{+}} \mu_{i, x}((\tau+t, \infty)) d t+ \\
& +v_{i} \int_{\mathscr{X}} N_{i}^{*}(d s) \int_{\mathscr{X}} Q_{i}(s, d u) \mu_{1-i, u}(\{0\}) \int_{\mathscr{X}} Q_{1-i}(u, d x) \int_{\mathscr{R}_{+}} \mu_{i, x}((\tau+t, \infty)) d t .
\end{aligned}
$$

Hence we obtain the relation

$$
v_{0}=\lim _{z \searrow 0} h_{0}(z)=v_{1} \int_{\mathscr{X}} N_{1}^{*}(d s) \int_{\mathscr{X}} Q_{1}(s, d x) \mu_{0, x}\left(\mathscr{R}_{+}\right) .
$$

By the definitions of the measures $N_{a}^{-}$and $N_{a}^{*}$ we have the relations

$$
\begin{gathered}
N_{0}^{*}(A) \int_{\mathscr{X}} N_{1}^{*}(d s) \int_{\mathscr{X}} Q_{1}(s, d x) \mu_{0, x}\left(\mathscr{R}_{+}\right)+ \\
\quad+\int_{A} \int_{\mathscr{X}} N_{1}^{*}(d s) Q_{1}(s, d x) \mu_{0, x}(\{0\})=N_{1}^{-}(A), \\
N_{1}^{*}(A)=N_{0}^{-}(A) .
\end{gathered}
$$

Using (19), (20), and the definition of the measures $N_{a}^{+}$we have

$$
1=v_{1}\left(\int_{\mathscr{X}} N_{0}^{+}(d s) m_{0, s}+\int_{\mathscr{X}} N_{1}^{+}(d s) m_{1, s}\right) \text {. }
$$

Substituting (19) into (18) and using (20) and (21) we obtain (7). Formulas (8) and (9) follow immediately from the potential theorem.

The following theorem gives the relations between the marginal measure $\tilde{N}_{a}(A \times B)=\bar{N}\left(A \times B \times \mathscr{R}_{+} \times\{a\}\right)$ and the measures $N_{a}^{-}$and $N_{a}^{+}$, $a=0,1$.

THEOREM 3. If $\bar{N}$ is a unique probability measure for the semi-group of operators $\left\{\overline{\mathscr{P}}_{t}, t \geqslant 0\right\}$ and if conditions (i)-(iv) are fulfilled, then the measures $N_{a}, N_{a}^{-}$, and $N_{a}^{+}$satisfy the relations

$$
\mathscr{A}(\tilde{\Pi}) \tilde{N}_{a}(A \times \mathscr{X})=v\left(N_{1-a}^{-}(A)-N_{a}^{+}(A)\right), \quad a=0,1,
$$

where $\tilde{\Pi}(t,(x, y, i), A \times B \times\{a\})=\bar{\Pi}\left(t, \bar{x}, A \times B \times \mathscr{R}_{+} \times\{a\}\right), a, i=0,1$, $t \geqslant 0, x, y \in \mathscr{X}, A, B \in \mathscr{F}$.

Proof. Let $f \in \overline{\mathscr{C}}_{0}$ and $f(\tilde{x})=h(x, y)$. Then we have

$$
\begin{aligned}
(23) \cdot & \frac{1}{t} \int_{\overline{\mathscr{X}}} \bar{N}(d \bar{x}) \int_{\mathscr{X}^{2} \times \mathscr{R}_{+}} \bar{\Pi}\left(t, \bar{x}, d \tilde{x}_{1} \times\{a\}\right) f\left(\tilde{x}_{1}\right)-\frac{1}{t} \int_{x^{2} \times \mathscr{R}_{+}} \bar{N}(d \tilde{x} \times\{a\}) f(\tilde{x}) \\
= & \frac{1}{t} \int_{\mathscr{X}^{2}} \bar{N}\left(d x \times d y \times \mathscr{R}_{+} \times\{a\}\right)\left[\int_{\mathscr{X}} P_{a, y}(t, x, d s) h(s, y)-h(x, y)\right]- \\
& -\frac{1}{t} \int_{\mathscr{X}^{2}} \bar{N}(d x \times d y \times(0, t] \times\{a\}) \int_{\mathscr{X}} P_{a, y}(t, x, d s) h(s, y) .
\end{aligned}
$$


By assumption (ii) and by (13), for every $\varepsilon>0$ there exists $\delta>0$ such that for $t<\delta$ we have

$$
\frac{1}{t} \int_{\mathscr{X}^{2}} \bar{N}(d x \times d y \times(0, t] \times\{a\})\left|\int_{\mathscr{X}} P_{a, y}(t, x, d s) h(s, y)-h(x, y)\right| \leqslant c \varepsilon .
$$

Therefore, from (i) and (9) we obtain

$$
\begin{aligned}
\lim _{t \rtimes 0} \frac{1}{t} \int_{\mathscr{X}^{2}} \bar{N}(d x \times d y \times(0, t] \times\{a\}) \int_{\mathscr{X}} P_{a, y}(t, x, d s) h(s, y) \\
=v \int_{\mathscr{X}} N_{a}^{+}(d x) \mu_{a, x}\left(\mathscr{R}_{+}\right) \int_{\mathscr{X}^{2}} \tilde{N}_{a}^{*}(d x \times d y) h(x, y) .
\end{aligned}
$$

The limit of the left-hand side of (23) for $t \searrow 0$ exists by lemma 2 and equals $\mathscr{A}(\bar{\Pi}) \bar{N}\left(A \times B \times \mathscr{R}_{+} \times\{a\}\right)$, hence by (24) there exists also the limit of the first expression on the right-hand side of (23) and it equals $\mathscr{A}(\tilde{\Pi}) \tilde{N}_{a}$. Using (7) we obtain

$$
\begin{aligned}
\mathscr{A}(\tilde{\Pi}) \tilde{N}_{a}(A \times B)= & v \int_{\mathscr{X}} N_{a}^{+}(d x) \mu_{a, x}\left(\mathscr{R}_{+}\right) \tilde{N}_{a}^{*}(A \times B)- \\
& -v \int_{\boldsymbol{A}} N_{a}^{+}(d x) I_{B}(x) \mu_{a, x}\left(\mathscr{R}_{+}\right) .
\end{aligned}
$$

By the definitions of the measures $\tilde{N}_{a}^{*}(A \times B)$ and $N_{a}^{-}(A)$ we have

$$
\begin{gathered}
\tilde{N}_{0}^{*}(A \times \mathscr{X}) \int_{\mathscr{X}} N_{0}^{+}(d x) \mu_{0, x}\left(\mathscr{R}_{+}\right)+\int_{\mathcal{A}} N_{0}^{+}(d x) \mu_{0, x}(\{0\})=N_{1}^{-}(A), \\
\tilde{N}_{1}^{*}(A \times \mathscr{X})=N_{0}^{-}(A) .
\end{gathered}
$$

Hence and from (25) we obtain (22).

In the case of a discrete state space, by theorem 3 we obtain theorem 1 from [9]. If $\mu_{0, y}(\{0\})=1$ for every $y \in \mathscr{X}$, then the process $\{X(t), t \geqslant 0\}$ is a piecewise Markov process and from theorems 2 and 3 we obtain the relations given in [7]. In particular, for a discrete state space we obtain also the relations included in [10] and [6].

\section{APPLICATIONS} Now we give some applications of the obtained results. We consider
the $M^{x} / G / 1$ queue with breakdown of the service line and the $M^{x} / G / 1$ queue with pre-emptive priority. 
We denote by small Jetters the Laplace-Stieltjes transforms of distribution functions and by the symbol ${ }^{\wedge}$ we denote the residual probability distribution, for example

$$
f(s)=\int_{\mathscr{R}_{0}} e^{-s x} d F(x), \quad \hat{F}(x)=\frac{\int_{(0, x]}(1-F(u)) d u}{\int_{\mathscr{R}_{+}}(1-F(u)) d u} .
$$

4.1. The system $M^{x} / G / 1$ with breakdown of the service line. Consider a single server system in which the input stream is a batched Poissonian stream with intensity $\lambda$ and with probability distribution $\left\{p_{j}, j=1,2, \ldots\right\}$ of the number of items in a batch. Consecutive service times are independent random variables with identical, continuous distribution functions $G(y)$ and with a finite expected value. Denote by $H(y)$ the distribution function of the batch service time; then we have

$$
H(y)=\sum_{j=1}^{\infty} p_{j} G^{* j}(y), \quad y \geqslant 0,
$$

where $G^{* j}(y), j=1,2, \ldots$, denotes the $j$-fold convolution of the distribution function $G(y)$. Additionally, we assume that both the breakdown times of the service line and its working times are independent random variables with distribution functions $F_{0}(y)$ and $F_{1}(y)$, and with finite expected values $m_{0}$ and $m_{1}$, respectively. The item with interrupted service continues its service after repairing the line.

In [5], p. 270-280, the steady-state conditions and probability distributions of the busy period of systems with breakdowns were investigated. In that paper the Laplace-Stieltjes transform of the distribution function of the virtual waiting time was given under the assumption that the breakdown may occur only in the idle period of the system or at any arbitrary moment but with an exponential probability distribution of working time. The system $M / G / 1$ with breakdown of the service line was considered also in [8], p. 85-101, and the Laplace-Stieltjes transform of the waiting time was there given.

In this section we give relations between the distribution function of the virtual waiting time and the distribution functions of the waiting time in breakdown and repair moments.

Let $X(t)$ denote the virtual waiting time at the moment $t$, and assume right-hand continuity of this process.

It is easy to remark that the process $\{X(t), t \geqslant 0\}$, valued in $\left(\mathscr{R}_{0}, \mathscr{B}_{0}\right)$, is an extended piecewise Markov process. The consecutive moments of breakdowns and repairs of the service line are regenerative moments of this process. In the working period of the line, $\left\{X(t), \tau_{2 m+1} \leqslant t<\tau_{2 m+2}\right\}$ 
is a Markov process with transition probabilities given by the formulas (see [11], p. 79)

$$
\begin{gathered}
P_{1}(t, x,[0, y])=K(t+y-x, t)-\int_{[0, t-x)} P_{1}(t-u, x,\{0\}) d K(u+y, u) \\
t, x, y \geqslant 0
\end{gathered}
$$

where

$$
K(y, t)=\sum_{n=0}^{\infty} e^{-\lambda t} \frac{(\lambda t)^{n}}{n !} B^{* n}(y)
$$

$$
d K(t+y, t)=\sum_{n=0}^{\infty} e^{-\lambda t} \frac{(\lambda t)^{n}}{n !} d_{t} H^{* n}(t+y),
$$

$$
P_{1}(t, x,\{0\})=\sum_{n=0}^{\infty} e^{-\lambda t} \frac{(\lambda t)^{n-1}}{n !} \int_{[0, t-x)} \lambda(t-u) d H^{* n}(u)
$$

with $H^{*_{0}}(y)=I_{[0, \infty)}(y)$.

In the breakdown period of the service line, $\{X(t), t \geqslant 0\}$ is a Markov process with transition probabilities given by the formula

$$
P_{0}(t, x,[0, y])=K(y-x, t), \quad t, x, y \geqslant 0 .
$$

Regenerative transitions are identity transitions, hence we have

$$
Q_{a}(x, A)=I_{A}(x), \quad x \geqslant 0, A \in \mathscr{B}_{0}, a=0,1 .
$$

Let us introduce the shorter notation

$$
N_{a}(y)=N_{a}([0, y]), \quad N_{a}^{-}(y)=N_{a}^{-}([0, y]), \quad a=0,1 .
$$

THEOREM 4. If in the system $M^{X} / G / 1$, with breakdown of the service line, the measure $\bar{N}$ is the stationary probability distribution for the extended Markov process $\{\bar{X}(t), t \geqslant 0\}$ and assumption (i) is fulfilled, then the distributions $N_{a}(y)$ and $N_{a}^{-}(y), a=0,1$, satisfy the relations

$$
\begin{gathered}
N_{0}(y)=\frac{v}{\lambda} \sum_{n=0}^{\infty} \int_{[0, y]}\left[N_{0}^{-}(y-x)-N_{1}^{-}(y-x)\right] d H^{* n}(x), \\
N_{1}(y)=[1-(1-v) \varrho] \sum_{n=0}^{\infty} \varrho^{n}(\hat{H})^{* n}(y)+ \\
+v \sum_{n=0}^{\infty} \varrho^{n} \int_{[0, y]}\left[\frac{1}{a_{1}} \hat{N}_{1}^{-}(y-x)-\frac{1}{a_{0}} \hat{N}_{0}^{-}(y-x)\right] d(\hat{H})^{* n}(x), \\
y \geqslant 0, \quad \frac{1}{v}=m_{0}+m_{1}, \quad \varrho=\frac{\lambda}{\mu}, \quad \frac{1}{\mu}=\int_{\mathscr{R}_{+}}(1-H(u)) d u, \\
\frac{1}{a_{i}}=\int_{\mathscr{R}_{+}}\left(1-N_{i}^{-}(x)\right) d x, \quad i=0,1 .
\end{gathered}
$$


Proof. We apply theorem 3 in the proof, thus we verify assumptions (ii)-(iv) of this theorem. Assumptions (iii) and (iv) are fulfilled by formula (31) and in view of the fact that the distribution functions of distances between consecutive regenerative moments are not dependent on the state of the process $\left\{X(t)_{x} t \geqslant 0\right\}$ at the regenerative moment. Since the transition probabilities defined by formulas (26)-(30) also do not depend on the state of the process $\{X(t), t \geqslant 0\}$ at the regenerative moment, to verify assumption (ii) it is sufficient to show that for any arbitrary function $f \in \mathscr{C}_{0}\left(\mathscr{R}_{0}\right)$ the expression

$$
\int_{\mathscr{R}_{0}} f(y) P_{a}(t, x, d y), \quad a=0,1,
$$

for $t \searrow 0$ tends to $f(x)$ uniformly on $\mathscr{R}_{0}$.

Let us denote by $\Delta(t, x, y)$ every expression which for $t \searrow 0$ tends to zero uniformly with respect to $x, y$.

For $a=0$ we have

$$
\int_{\mathscr{R}_{0}} f(y) P_{0}(t, x, d y)=f(x) I_{[0, \infty)}(x) e^{-\lambda t}+\Delta(t, x) \rightarrow f(x) \quad \text { as } t \backslash 0
$$

uniformly on $\mathscr{R}_{0}$.

For $a=1$ we show now that, for $y>0$ and $x \geqslant 0$,

$$
\int_{[0, t-x)} P_{1}(t-u, x,\{0\}) d K(u+y, u)=\Delta(t, x, y) \text {. }
$$

Using formulas (27)-(29) we obtain

$$
\begin{gathered}
\int_{[0, t-x)} P_{1}(t-u, x,\{0\}) d K(u+y, u)=e^{-\lambda t} \int_{[0, t-x)} d_{u} I_{[0, \infty)}(u+y)+ \\
+e^{-\lambda t} \int_{[0, t-x)}\left(\int_{[0, t-u-x)} \lambda(t-u-v) d H(v)\right) d_{u} I_{[0, \infty)}(u+y)+ \\
+e^{-\lambda t} \int_{[0, t-x)} \lambda u d_{u} H(u+y)+o(t) .
\end{gathered}
$$

The first two components on the right-hand side of (34) are equal to zero, the third one is $\Delta(t, x, y)$. Hence for $a=1$ we have

$$
\int_{\mathscr{R}_{0}} f(y) P_{1}(t, x, d y)=e^{-\lambda t} f\left((x-t)_{+}\right)+\Delta(t, x) \rightarrow f(x) \quad \text { as } t \searrow 0
$$

uniformly on $\mathscr{R}_{0}$, where $(a)_{+}$denotes $\max (a, 0)$.

In order to use (22) we compute $\mathscr{A}(\tilde{\Pi}) N_{a}(y), y \geqslant 0, a=0,1$. Hence we have

$$
\tilde{\Pi}(t,(x, i),[0, y] \times\{a\})=P_{i}(t, x,[0, y]) \delta_{i, a}, \quad a, i=0,1, x, y, t \geqslant 0
$$

Then we obtain

$$
\mathscr{A}(\tilde{\Pi}) N_{0}(y)=-\lambda N_{0}(y)+\lambda \int_{\mathscr{R}_{0}} H(y-x) d N_{0}(x), \quad y \geqslant 0 .
$$


From (34), by the continuity of the distribution function $H(y)$, it follows that

$$
\int_{[0, t-x)} P_{1}(t-u, x,\{0\}) d K(u+y, u)=o(t), \quad y>0, x \geqslant 0 .
$$

Hence we have

$$
\mathscr{A}(\tilde{\Pi}) N_{1}(y)=\frac{d}{d y} N_{1}(y)-\lambda N_{1}(y)+\lambda \int_{\mathscr{R}_{0}} H(y-x) N_{1}(d x), \quad y>0 .
$$

By (35), (36), and (22) we have

$$
-\lambda N_{0}(y)+\lambda \int_{\mathscr{R}_{0}} H(y-x) d N_{0}(x)=v\left[N_{1}^{-}(y)-N_{0}^{-}(y)\right], \quad y \geqslant 0,
$$

$$
\frac{d}{d y} N_{1}(y)-\lambda N_{1}(y)+\lambda \int_{\mathscr{R}_{0}} H(y-x) d N_{1}(x)=v\left[N_{0}^{-}(y)-N_{1}^{-}(y)\right], \quad y>0 .
$$

Using Laplace-Stieltjes transforms in (37) and (38), for $\operatorname{Res}>0$ we obtain

$$
\begin{gathered}
n_{0}(s)=\frac{v\left[n_{0}^{-}(s)-n_{1}^{-}(s)\right]}{\lambda[1-h(s)]} \\
n_{1}(s)=\frac{v\left[n_{0}^{-}(s)-n_{1}^{-}(s)\right]+s N_{1}(0)}{s-\lambda[1-h(s)]} .
\end{gathered}
$$

Applying formula (13) we compute the constant $N_{0}(0)$ and we have

$$
N_{0}(0)=\frac{v}{\lambda} N_{0}^{-}(0)\left[1-f_{0}(\lambda)\right]
$$

Taking in (40) the limit for $s \searrow 0$ we compute the constant $N_{1}(0)$. We have

$$
N_{1}(0)=1-(1-v) \varrho .
$$

Inversion of the Laplace-Stieltjes transforms in formulas (39)-(42) gives (32) and (33).

Applying theorem 2 (b) we obtain the following corollary:

Corollary 2. The distribution functions $N_{a}(y)$ and $N_{a}^{-}(y), a=0,1$, for $y \geqslant 0$ fulfil the relations

$$
\begin{gathered}
N_{0}(y)=v \int_{\mathscr{R}_{+}} e^{-\lambda t}\left(1-F_{0}(t)\right) \sum_{n=0}^{\infty} \frac{(\lambda t)^{n}}{n !} \int_{\mathscr{R}_{0}} H^{* n}(y-x) d N_{0}^{-}(x) d t, \\
N_{1}(y)=v \int_{\mathscr{R}_{+}}\left(1-F_{1}(t)\right) \int_{\mathscr{R}_{0}} P_{1}(t, x,[0, y]) d N_{1}^{-}(x) d t .
\end{gathered}
$$


Relation (44) is practically not very useful because of the complicated form of the transition probabilities $P_{1}(t, x,[0, y])$.

4.2. The $M^{X} / G / 1$ system with pre-emptive priority. Formulas (32), (33), (43), and (44) become effective if we find the form of the distribution function $N_{0}^{-}(y)$ for the Markov chain imbedded at the breakdown moments of the service line. In every particular case this is possible, since the chain is completely described, but numerical difficulties may arise. Now we give an example of a system in which the Laplace-Stieltjes transform $n_{0}^{-}(s)$ of the distribution function may be calculated effectively.

Let $\lambda_{0}$ and $\lambda$ be intensities of the batched Poissonian input stream of the calls of priority and non-priority items to the system, respectively, and let $\left\{q_{j}, j=1,2, \ldots\right\}$ and $\left\{p_{j}, j=1,2, \ldots\right\}$ be probability distributions of the number of items in a batch of priority and non-priority items, respectively. We assume that consecutive services of items are independent random variables with identical, continuous distribution functions $G_{0}(y)$ and $G(y)$ and with finite expected values. In the considered system we analyze the virtual waiting time $\{X(t), t \geqslant 0\}$ of nonpriority items.

The described system may be considered as the system $M^{\boldsymbol{x}} / G / 1$ with breakdown of the line caused by priority items in the system. By such a treatment the distribution function of the working time of the line is equal to $1-e^{-\lambda_{0} y}, y \geqslant 0$, and the distribution function of the breakdown time of the line $F_{0}(y)$ is the well-known distribution function of the busy period in the system $M / G / 1$ (see [11], p. 70) in which the input stream is Poissonian with intensity $\lambda_{0}$ and which has the distribution function of the service line of the form

$$
\sum_{j=1}^{\infty} q_{j} G_{0}^{* j}(y), \quad y \geqslant 0 .
$$

Thus relations (32), (33), (43), and (44) may be applied also to this system. Now we find the Laplace-Stieltjes transform $n_{0}^{-}(s)$.

Denote by $\left\{p_{j}^{* i}, j=0,1, \ldots\right\}$ the probability distribution of the number of non-priority items in $i$ batches, $i=0,1, \ldots$, where $p_{j}^{*_{0}}=\delta_{j, 0}$, and let

$$
\begin{gathered}
u_{j}=\int_{\mathscr{R}_{0}} e^{-\lambda t} d F_{0}(t) \sum_{i=0}^{j} \frac{(\lambda t)^{i}}{i !} p_{j}^{* i}, \quad j \geqslant 0, \\
w_{j}=\frac{\lambda p_{j}+\lambda_{0} u_{j}}{\lambda+\lambda_{0}\left(1-u_{0}\right)}, \quad j \geqslant 1, \\
H_{0}(y)=\sum_{j=0}^{\infty} u_{j} G^{* j}(y), \quad H_{1}(y)=\sum_{j=1}^{\infty} w_{j} G^{* j}(y), \quad y \geqslant 0 .
\end{gathered}
$$


TheOREM 5. In the system $M^{x} / G / 1$ with pre-emptive priority the Laplace-Stieltjes transform $n_{0}^{-}(s)$ of the distribution function $N_{0}^{-}(y)$ of the stationary waiting time at the priority-item-arrival moments is given by the formula

$$
n_{0}^{-}(s)=\frac{n^{\prime}(s)[s-\lambda(1-h(s))]-s\left(1-\varrho-\varrho_{0}\right)}{1-h_{0}(s)}, \quad \operatorname{Re} s>0
$$

where

$$
\begin{array}{r}
n^{\prime}(s)=\frac{\left(1-\varrho_{1}\right) s}{s-\lambda_{1}\left(1-h_{1}(s)\right)}, \quad \varrho_{i}=\frac{\lambda_{i}}{\mu_{i}}, \quad \frac{1}{\mu_{i}}=\int_{\mathscr{R}_{0}}\left(1-H_{i}(u)\right) d u, \\
i=0,1, \lambda_{1}=\lambda+\lambda_{0}\left(1-u_{0}\right) .
\end{array}
$$

Proof. Introduce a new time variable $t^{\prime}$ contracting to points the busy periods of the line with service of priority items. At the moment $t^{\prime}$ the input stream of non-priority items is a mixture of both a batched Poissonian input stream with intensity $\lambda$ and with the probability distribution $\left\{p_{j}, j=1,2, \ldots\right\}$ of the number of items in a batch and of a batched Poissonian input stream with intensity $\lambda_{0}$ and with the probability distribution $\left\{u_{j}, j=0,1, \ldots\right\}$ of the number of items in a batch. Omitting empty batches we obtain a batched Poissonian input stream with intensity $\lambda_{1}$ and with the probability distribution $\left\{w_{j}, j=1,2, \ldots\right\}$.

In order to investigate the probability distribution of the stationary virtual waiting time $\left\{X^{\prime}\left(t^{\prime}\right), t^{\prime} \geqslant 0\right\}$, the considered system may be treated as the system $M / G / 1$ with a Poissonian input stream with intensity $\lambda_{1}$ and with the distribution function of the service time $H_{1}(y)$. Denoting by $N^{\prime}(y)$ the distribution function of the virtual waiting time, we have (see [11], p. 44, or [5], p. 243)

$$
n^{\prime}(s)=\frac{\left(1-\varrho_{1}\right) s}{s-\lambda_{1}\left(1-h_{1}(s)\right)}, \quad \operatorname{Re} s>0
$$
Now we find the relation between transforms $n_{0}^{-}(s)$ and $n^{\prime}(s)$. Let
$\left\{\tau_{m}^{\prime}, m=0,1, \ldots\right\}$ denote the sequence of consecutive arrivals of the batches in the batched Poissonian input stream with intensity $\lambda_{0}$ and with the probability distribution $\left\{u_{j}, j=0,1, \ldots\right\}$ of the number of items in a batch. It is easy to notice that the equality

$$
\operatorname{Pr}\left(X^{\prime}\left(\tau_{m}^{\prime}-0\right) \leqslant y\right)=N_{0}^{-}(y), \quad y \geqslant 0
$$

holds. The process $\left\{X^{\prime}\left(t^{\prime}\right), t^{\prime} \geqslant 0\right\}$ is a piecewise Marko $\nabla$ process with regenerative moments $\left\{\tau_{m}^{\prime}, m=0,1, \ldots\right\}$ (see [7]). In the intervals $\tau_{m}^{\prime}<t^{\prime}$ 
$<\tau_{m+1}^{\prime}, m=0,1, \ldots$, the process $\left\{X^{\prime}\left(t^{\prime}\right), t^{\prime} \geqslant 0\right\}$ is a Markov process with transition probabilities given by formulas (26)-(29). Applying theorem 6 from [7], analogous to theorem 3 from this paper, we obtain the relation

$$
\begin{aligned}
\frac{d}{d y} N^{\prime}(y)-\lambda N^{\prime}(y) & +\lambda \int_{\mathscr{R}_{0}} H(y-x) d N^{\prime}(x) \\
= & \lambda_{0}\left(N_{0}^{-}(y)-\int_{\mathscr{R}_{0}} H_{0}(y-x) d N_{0}^{-}(x)\right), \quad y>0 .
\end{aligned}
$$

Using the Laplace-Stieltjes transform in (46) we obtain (45).

\section{References}

[1] J. Azema, M. Duflo and D. Revuz, Mesure invariante des processus de Markov récurrents, Lect. Notes Math. 88 (1968), p. 22-33.

[2] P. Billingsley, Convergence of probability measures, New York 1968.

[3] E. B. Dynkin (Е. Б. Дынкин), Марковские прочессы, Москва 1963.

[4] I. I. Gikhman and A. V. Skorokhod (И.И. Гихман и А. В. Скороход), Теория случайных прочессов, I, Москва 1971.

[5] B. W. Gniedenko and N. I. Kowalenko, Wstep do teorii obstugi masowej, Warszawa 1966.

[6] M. Jankiewicz and B. Kopociński, Steady-state distributions of piecewise Markov processes, Zastosow. Matem. 15 (1976), p. 25-32.

[7] M. Jankiewicz and T. Rolski, Piecewise Markov processes on a general state space, ibidem 15 (1977), p. 421-436.

[8] G. P. Klimov (Г. П. Климов), Стохастические системь обслужсиания, Москва 1966.

[9] I. Kopocińska, Uogólnione procesy przedzialami markowskie, Matem. Stosow. 9 (1977), p. 117-122.

[10] A. Kuczura, Piecewise Markov processes, SIAM J. Appl. Math. 24 (1973), p. 169-181.

[11] N. U. Prabhu, Queues and inventories, New York 1965.

[12] Z. Semadeni, Banach spaces of continuous functions, I, Warszawa 1971.

[13] R. L. Tweedie, Sufficient conditions for ergodicity and recurrence of Markov chains on a general state space, Stoch. Processes Appl. 3 (1975), p. 385-403. 
MARIA JANKIE WICZ (Wroclaw)

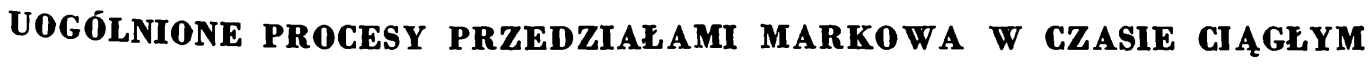

\author{
ST RESZCZENIE
}

W pracy [9] zostały wprowadzone uogólnione procesy przedziałami Markowa z dyskretna przestrzenią stanów.

W tej pracy bada się uogólnione procesy przedziałami Markowa z ogólną przestrzenia stanów oraz pewne ich zastosowania. Stosując metode rozszerzania procesu do procesu Markowa oraz twierdzenia teorii półgrup operatorów kontrakcji, podaje się związki między stacjonarnym rozkładem prawdopodobieństwa stanów. procesu i stacjonarnymi rozkładami pewnych włożonych łańcuchów Markowa. 